\title{
VLBI Observations of SNR in M82 - Is 41.95+575 an Anomalous SNR or Something Else ?
}

\author{
T.W.B.Muxlow, A. Pedlar, A.R. McDonald,, S.T. Garrington, W.M. \\ Trotman, P.N. Wilkinson, P.J. Diamond \\ Jodrell Bank Observatory, University of Manchester \\ M.A. Garrett(1), K.A. Wills(2) \\ Joint Institute for VLBI in Europe(1), University of Sheffield(2)
}

\begin{abstract}
Global VLBI 1.7GHz datasets from 1990 and 1998 have been used to study the evolution of SNR $41.95+575$ in M82. An expansion velocity of around $1800 \mathrm{~km} / \mathrm{s}$ is found with an implied birth around 1915 . A young $41.95+575$ would resemble radio supernovae seen in Arp220, although it could also be an evolving accretion disc system
\end{abstract}

\section{The Nature of $41.95+575$}

Global VLBI L-Band datasets from September 1990 and November 1998 have been used to study the evolution of the SNR $41.95+575$ in the nearby $(\mathrm{d}=3.2 \mathrm{Mpc})$ starburst galaxy M82. The radio structure has evolved between these epochs and the separation of the peaks in the NE and SW components has been found to have increased by 1.9 mas. Assuming linear motion, this gives an expansion velocity of $1800 \pm 200 \mathrm{~km} / \mathrm{s}$ with an implied zero size birth date of around 1915 . This velocity is about 5 times lower than that measured for a number of more conventional shell-like SNR in M82. The flux density of $41.95+575$ has been monitored for many years (eg Kronberg et al, 2000) and is seen to be decreasing at $8.5 \%$ per year (Trotman, 1996). In its youth, $41.95+575$ would resemble the extremely luminous radio supernovae detected in Arp220 which are thought to be of a class of which RSN 1986J is a prototype. We thus propose that SNR $41.95+575$ is anomalous and differs from the 50 more conventional young SNR seen in M82 which resemble shells or partial shells with expansion velocities around $10,000 \mathrm{~km} / \mathrm{s}$ (Pedlar et al, 1999). Alternatively, $41.95+575$ may not be an SNR, but may be associated with a stellar accretion disc system decaying from some recent episodic activity.

\section{References}

Kronberg, P.P., Sramek, R.A., Birk, G.T., Dufton, Q.W., Clarke, T.E., \& Allen, M.L., 2000,ApJ, 535,706 .

Pedlar, A., Muxlow, T.W.B., Garrett, M.A., Diamond, P.J., Wills, K.A., Wilkinson, P.N., \& Alef, W., 1999, MNRAS, 307, 761.

Trotman, W.M., 1996, Ph.D. Thesis, University of Manchester. 

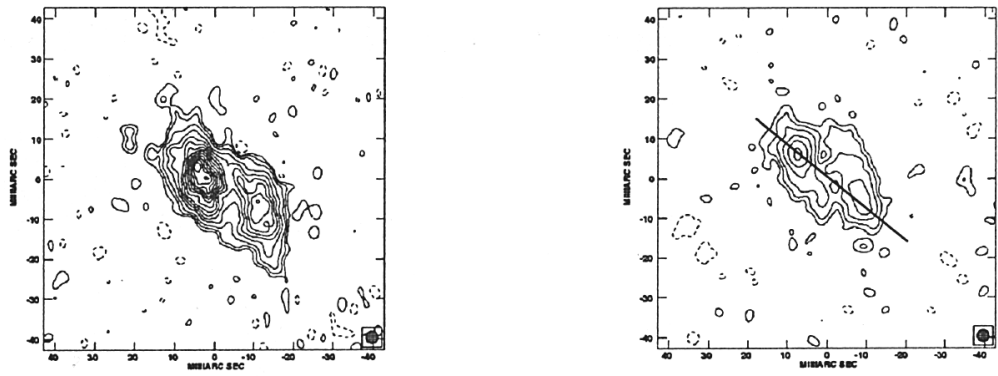

Figure 1 (left) and Figure 2 (right). Global VLBI $20 \mathrm{~cm}$ radio images of the compact SNR 41.95+575 in M82 from epochs September 1990 and November 1998 respectively. $\mathrm{CI}=-1,1,2,4,8,12 \ldots 80 \times 0.11 \mathrm{mJy} /$ beam ( 3 mas circular). The position of the brightness profile shown in Figure 3 is plotted on Figure 2.

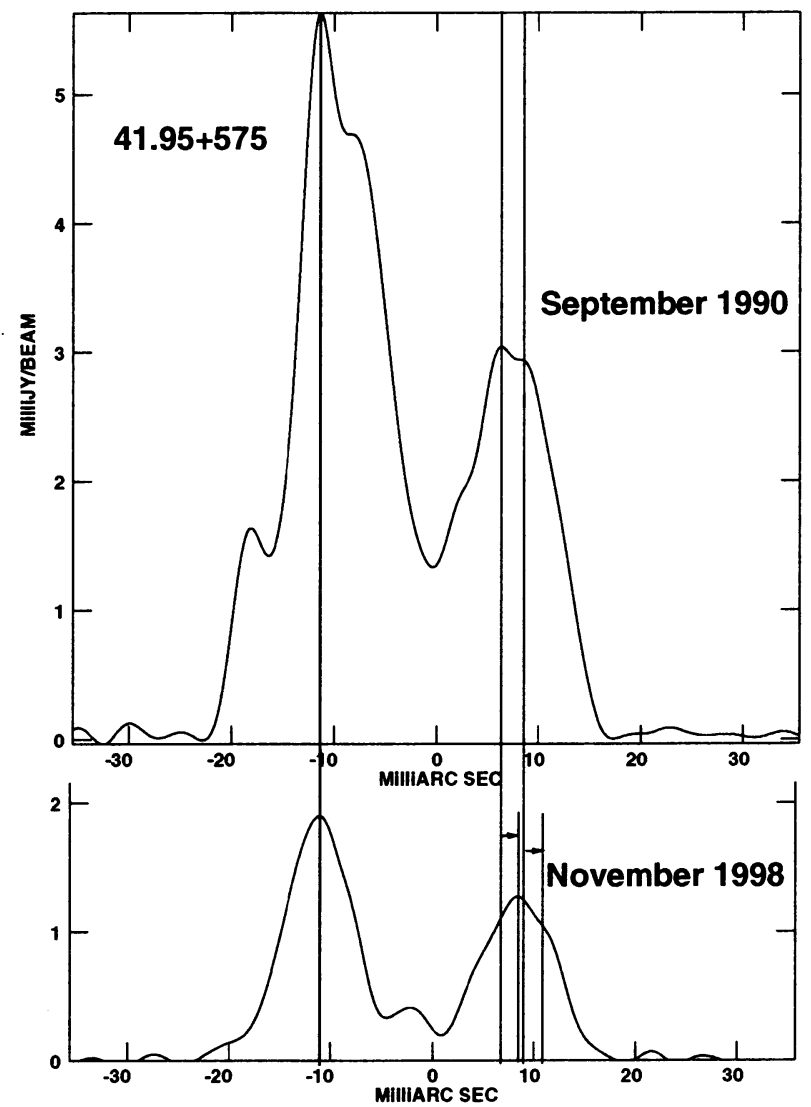

Brightness profiles through the 1990 and 1998 global VLBI images Figure 3 